\title{
Nanocompósitos de Poliamida 6 e Argila Organofílica: Estudo da Cristalinidade e Propriedades Mecânicas
}

\author{
Marcelo F. L. de Oliveira \\ Departamento de Química Orgânica, UERJ \\ Divisão de Processamento e Caracterização de Materiais, INT \\ Márcia G. de Oliveira \\ Divisão de Processamento e Caracterização de Materiais, INT \\ Márcia C. A. M. Leite \\ Departamento de Química Orgânica, UERJ
}

Resumo: Nanocompósitos de poliamida 6 e argila organofílica claytone 40 foram preparados por intercalação por fusão, utilizando misturador de câmara interna equipado com rotores do tipo Roller. A adição de teores crescentes de claytone 40 na matriz de PA6 foi avaliada quanto ao grau de dispersão, cristalinidade, propriedades térmicas e as propriedades mecânicas. As composições obtidas foram caracterizadas por difração de raios X (DRX), calorimetria diferencial de varredura (DSC), microscopia eletrônica de varredura (MEV) e propriedades mecânicas. Os difratogramas e as micrografias sugeriram a ocorrência de formação de estruturas parcialmente esfoliadas e/ ou intercaladas, fato que foi associado com o aumento nos valores de tensão e o módulo elástico. A comparação entre os resultados de DSC e DRX das misturas revelaram alterações estruturais na cristalinidade em relação à PA6 correlacionando a cristalinidade à variação nas propriedades mecânicas.

Palavras-chave: Nanocompósitos, montmorilonita, argila organofílica, poliamida 6.

\section{Nanocomposites of Polyamide 6 and Organoclay: Crystallinity and Study of Mechanical Properties}

\begin{abstract}
Nanocomposites of polyamide 6 and Claytone 40 organoclay were prepared with the melt intercalation technique using an internal mixer chamber coupled with roller type rotors. The addition of increasing amounts of Claytone 40 into the PA6 matrix was evaluated for the degree of dispersion, crystallinity, thermal and mechanical properties. The compositions were characterized with X-ray diffraction (XRD), differential scanning calorimetry (DSC), scanning electron microscopy (SEM) and mechanical properties. The diffraction patterns and microscope images suggest the formation of partially exfoliated or intercalated structures, which was associated with an increase in stress values and elastic modulus. The comparison of DSC and XRD results from the mixtures revealed changes in the behavior of crystalline structures, thus indicating that the PA6 crystallinity is correlated with the mechanical properties.
\end{abstract}

Keywords: Nanocomposites, montmorillonite, organoclays, polyamide 6.

\section{Introdução}

Nanocompósitos de polímeros e silicatos em camadas são materiais que representam uma alternativa muito vantajosa em relação aos compósitos de polímeros com cargas convencionais. Silicatos como a montmorilonita têm sido extensivamente reportado na literatura como cargas reforçantes de matrizes poliméricas por suas caracterísitcas geométricas: dimensões da ordem do nanômetro e de elevada razão de aspecto e área superficial. O emprego de quantidades mínimas desses silicatos (menor do que 10\%) como fase dispersa em polímeros já são suficientes para promover aumento nas propriedades térmicas, mecânicas, de barreira, inflamabilidade e estabilidade dimensional dos nanocompósitos quando comparados aos compósitos convencionais que requerem uma quantidade de 30 a $40 \%$ de reforço ${ }^{[1-6]}$.

Devido às características hidrofílicas da montmorilonita, pouca dificuldade deve ser encontrada no desenvolvimento de nanocompósitos com polímeros polares, principalmente se a superfície dessa argila sofrer tratamentos com íons alquilamônio tornando-a organofílica. Dessa maneira, ocorre aumento da interação da carga com o polímero, melhorando a sua molhabilidade e facilitando a penetração das moléculas orgânicas entre as camadas da $\operatorname{argila~}^{[1-6]}$.
Poliamida 6 (PA6) é um polímero usado em diversas aplicações na área da engenharia devido às suas propriedades versáteis tais como, elevado módulo elástico e boa estabilidade dimensional à temperaturas elevadas dentre outras ${ }^{[7-9]}$. De acordo com a literatura, possui três estruturas cristalinas $(\alpha, \gamma$ e $\beta)$. $\mathrm{Na}$ PA6 é predominante a forma cristalina $\alpha$ e menos do que $3 \%$ das formas cristalinas estão no arranjo $\gamma^{[8]}$. A estrutura cristalina da PA6 depende da história térmica, da presença de carga, das condições de processamento e de cristalização. Entretanto, ambas as formas cristalinas podem coexistir na PA6, porém a forma cristalina $\alpha$ é a mais estável termodinamicamente ${ }^{[8]}$.

O objetivo desse trabalho é avaliar os efeitos da dispersão de argila organofílica na matriz da PA6 realizadas por intercalação no estado fundido. DSC e DRX são utilizados para caracterizar a natureza cristalina e o grau de cristalinidade. As propriedades mecânicas desses nanocompósitos serão avaliadas e relacionadas à morfologia dos mesmos. A ênfase será sobre a cristalinidade e as propriedades mecânicas das amostras injetadas relacionando com o tipo de estrutura formado e a morfologia. 


\section{Experimental}

\section{Materiais}

PA6 (MAZMID B260, índice de fluidez = 22-35 g / 10 minutos - ISO 1133, densidade 1,14 g.cm ${ }^{-3}$ ) foi adquirida junto a Mazzaferro Indústria Comércio de Polímeros e Fibras Ltda. Montmorilonita modificada organicamente com cloreto de dimetildioctadecil amônio (Claytone ${ }^{\circledR}$ 40) contendo 26,64\% em massa de sal e densidade $1,6 \mathrm{~g} . \mathrm{cm}^{-3}$ foi adquirida junto a BUNTECH Tecnologia em insumos Ltda. Irganox $245 \circledR$, um composto fenólico inodoro de baixa volatilidade e densidade $1,14 \mathrm{~g} . \mathrm{cm}^{-3}$, foi gentilmente cedido pela Ciba ${ }^{\circledR}$ Specially Chemicals Corporation do Brasil e utilizado como antioxidante para a fase da poliamida.

\section{Preparação dos nanocompósitos}

Todas as misturas foram preparadas por intercalação no estado fundido a $220^{\circ} \mathrm{C}$ em câmara interna de mistura acoplada ao reômetro de torque, modelo Haake Polylab OS Rheodrive 4 utilizando rotores do tipo ROLLER, a $80 \mathrm{rpm}$ por 5 minutos. PA6 e argila organofílica foram previamente secas em estufa a vácuo, na temperatura de $80{ }^{\circ} \mathrm{C}$ por 24 horas antes do processamento. A quantidade de argila empregada na fase da PA6 variou em 2,5; 5,0; 7,5 e $10 \mathrm{phr}$.

\section{Caracterização}

\section{Difração de raios X (DRX)}

Os difratogramas da argila modificada e de cada amostra foram obtidos em um difratômetro de raios $\mathrm{X}$ com radiação $\mathrm{K} \alpha$ do cobre (comprimento de onda $\lambda=1,5418 \AA$ ), tensão de $40 \mathrm{kV}$, corrente de $30 \mathrm{~mA}$, passo de $0,01^{\circ} / \mathrm{min}$. Foram realizadas duas análises para cada amostra em diferentes faixas de varredura. A primeira faixa de varredura entre $2 \theta$ de 1,5 a $12^{\circ}$, para avaliar a variação da distância interplanar $\left(\mathrm{d}_{001}\right)$ calculada pela equação de Bragg. A segunda faixa de varredura foi realizada entre $2 \theta$ de 10 a $50^{\circ}$ para verificar o comportamento da cristalinidade dos nanocompósitos.

\section{Propriedades térmicas}

O comportamento das temperaturas de fusão, de cristalização e o grau de cristalinidade dos nanocompósitos foram avaliados por calorimetria diferencial de varredura (DSC) em equipamento TA Instruments, modelo DSC Q-100. As condições de teste foram: fluxo de nitrogênio a $30 \mathrm{~mL} / \mathrm{min}$; aquecimento com taxa de $10{ }^{\circ} \mathrm{C} / \mathrm{min}$ entre 70 a $250{ }^{\circ} \mathrm{C}$ de temperatura; isoterma a $250{ }^{\circ} \mathrm{C}$ por 5 minutos; resfriamento até $70{ }^{\circ} \mathrm{C}$ com taxa de $10{ }^{\circ} \mathrm{C} / \mathrm{min}$ e por fim novo aquecimento nas mesmas condições do primeiro. Os termogramas de fusão foram analisados após a segunda corrida de aquecimento. $\mathrm{O}$ grau de cristalinidade $\left(\mathrm{X}_{\mathrm{c}}\right)$ dos nanocompósitos foi calculado a partir da razão da entalpia de fusão da poliamida 6 presente no nanocompósito pela entalpia de fusão da poliamida 6 $100 \%$ cristalina $\left(\Delta \mathrm{H}_{\text {nano }} / \Delta \mathrm{H}_{\mathrm{PA} 6}{ }^{\circ}\right)$, onde $\Delta \mathrm{H}_{\mathrm{PA} 6}=190,8 \mathrm{~J} \cdot \mathrm{g}^{-1[10]}$.

\section{Propriedades mecânicas}

Os corpos de prova para ensaio de tração foram obtidos segundo a norma DIN 53504 em injetora Battenfleld ${ }^{\mathrm{TM}}$, modelo Plus 35, com $11 \mathrm{MPa}$ de pressão de fechamento de molde, $260^{\circ} \mathrm{C}$ de temperatura de injeção, $9 \mathrm{MPa}$ de pressão de injeção e $25^{\circ} \mathrm{C}$ de temperatura de molde. Os ensaios foram feitos em máquina universal de ensaios EMIC, modelo DL-2000, com célula de carga de $1 \mathrm{KN}$, velocidade de separação entre as garras de $10 \mathrm{~mm} / \mathrm{min}$, a temperatura de $23 \pm 0,3{ }^{\circ} \mathrm{C}$ e umidade relativa de $70 \pm 0,5 \%$. Os resultados foram obtidos a partir da média de seis corpos de prova.

\section{Microscopia eletrônica de varredura (MEV)}

A morfologia dos nanocompósitos foi investigada através das imagens dos corpos de prova criofraturados em nitrogênio líquido.
As amostras foram recobertas com carbono e visualizadas em um microscópio eletrônico de varredura FEI Company, modelo INSPECT S50 com voltagem de $25 \mathrm{kV}$, utilizando os seguintes detectores: elétrons secundários (SEI) para investigar a topografia dos materiais e EDS para mapeamento de silício (Si).

\section{Resultados e Discussão}

\section{Difração de raios $X(D R X)$}

A Figura 1 apresenta as curvas de DRX referentes à argila modificada claytone 40 e aos nanocompósitos de PA6 com diferentes teores de argila variando entre 0 e $10 \mathrm{phr}$.

A argila Claytone 40 apresentou dois picos de difração: o primeiro em ângulo de difração na região de $7,16^{\circ}$, correspondente à montmorilonita original e espaçamento basal de $1,23 \mathrm{~nm}$; o segundo pico em ângulo de difração na região de $2,95^{\circ}$, relativo à montmorilonita intercalada com o agente surfactante e espaçamento basal de 2,99 $\mathrm{nm}$. Esse resultado é semelhante ao da literatura ${ }^{[11]}$.

Em relação às misturas, pode-se observar que as curvas de DRX demonstram perda gradual do pico característico da monmorilonita com a redução do teor de argila. Para a amostra com 2,5 phr de claytone 40 , não foi possível observar o pico característico da montmorilonita.

Esse resultado pode ser atribuído tanto à desarticulação do empilhamento das camadas da argila (possível esfoliação), quanto ao grau de diluição da nanocarga, principalmente para o teor de 2,5 $\mathrm{phr}^{[12,13]}$.

A Tabela 1 mostra os valores do espaçamento basal dos nanocompósitos de PA6. Quanto aos valores das distâncias interplanares dos nanocompósitos com teores de 5,0; 7,5 e $10 \mathrm{phr}$ observaram-se aumento nas distâncias interlamelares em relação à argila modificada, o que indica a ocorrência de intercalação das cadeias de PA6 entre as lamelas da montmorilonita.

Para complementar os resultados obtidos a partir dos difratogramas ilustrados na Figura 1, a dispersão da argila na matriz de PA6 foi investigada por MEV. As Figuras 2a-d e 2e-h ilustram as

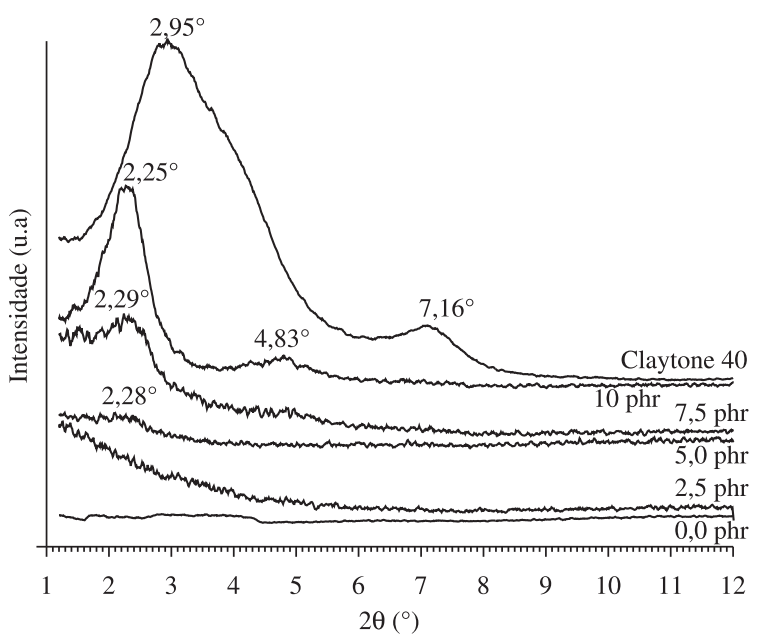

Figura 1. DRX da argila modificada claytone 40, da PA6 e dos nanocompósitos de poliamida com teores crescentes de argila modificada.

Tabela 1. Resultados de difração de raios $X$ (picos característicos e espaçamento basal) dos nanocompósitos de PA6-Claytone 40.

\begin{tabular}{ccc}
\hline Clay 40 (\%) & $\mathbf{2 \theta}$ & $\mathbf{d}(\mathbf{0 0 1}) \mathbf{n m}$ \\
\hline 5,0 & 2,25 & 3,93 \\
7,5 & 2,29 & 3,86 \\
10,0 & 2,28 & 3,87 \\
\hline
\end{tabular}




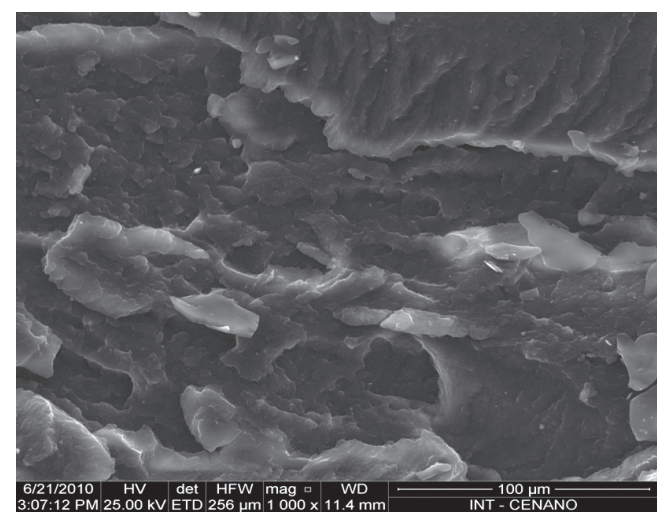

(a)

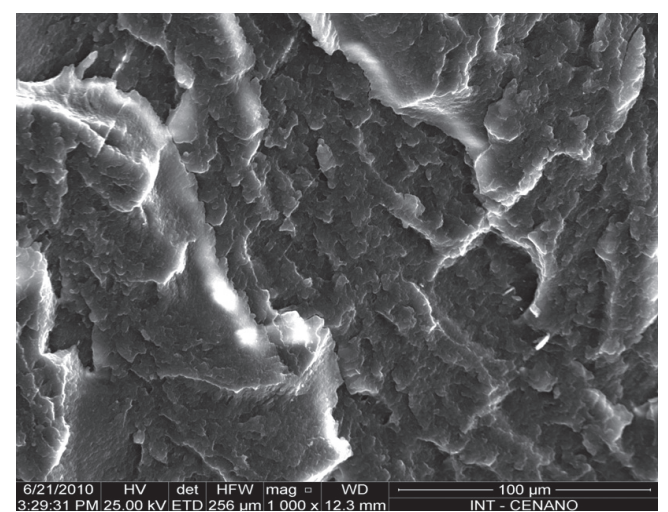

(b)

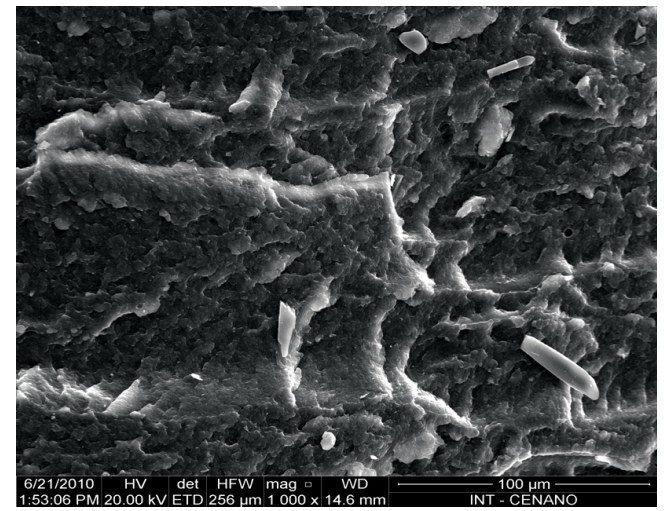

(c)

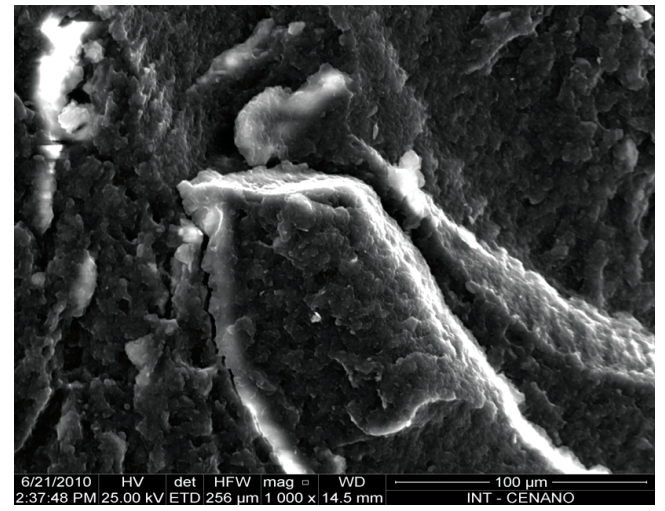

(d)

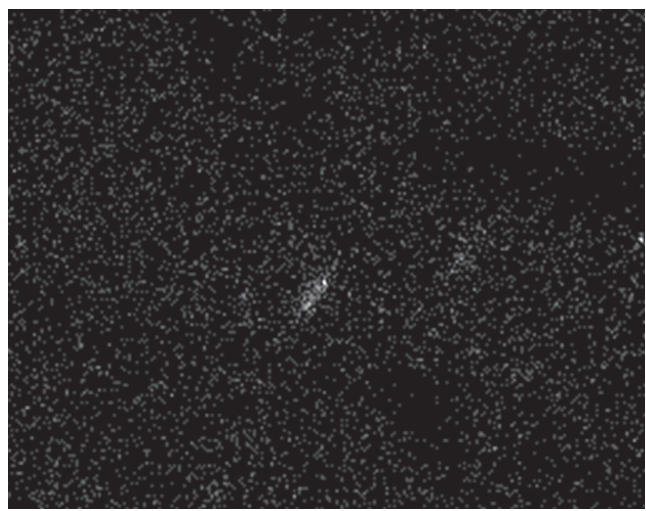

(e)

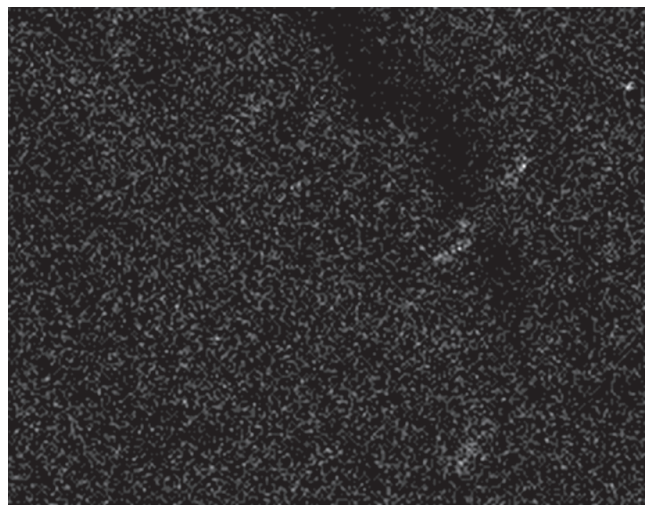

(f)

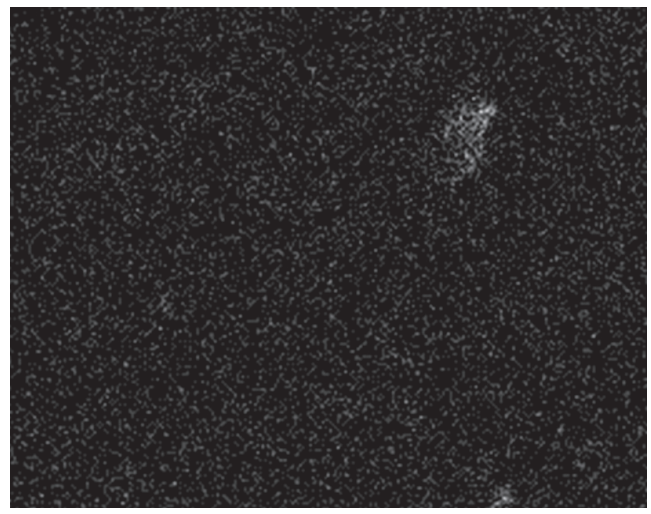

(g)

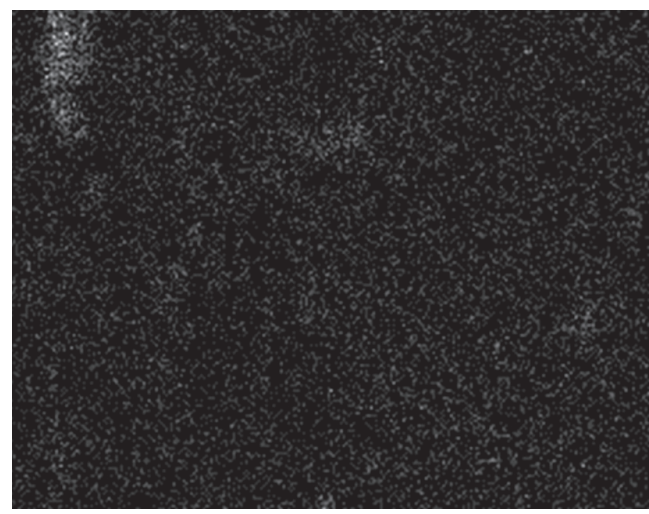

(h)

Figura 2. Micrografias dos nanocompósitos de PA6 com teores crescentes de argila. Com detector ES: a) 2,5 phr; b) 5,0 phr; c) 7,5 phr e d) 10 phr. Com detector EDS para mapeamento de Si: e) 2,5 phr; f) 5,0 phr; g) 7,5 phr e h) $10 \mathrm{phr}$. 
micrografias dos nanocompósitos de PA6 com teores crescentes de claytone 40, utilizando como detector, o de elétrons secundários e EDS respectivamente.

A Figura 2a não apresenta contraste que possa ser relacionado com a presença da nanocarga. No entanto, nas demais Figuras $2 b-d$, as imagens mostraram pontos claros espalhados na amostra que pode ser atribuído à presença da nanocarga.

As Figuras 2e-hilustram as imagens dos mesmos nanocompósitos com detector de EDS para mapeamento do Si e avaliação da dispersão da argila. Os pontos claros indicam a localização do elemento silício. Como esperado, esses pontos se intensificam com o aumento do teor de carga. No entanto, nas Figuras $2 \mathrm{~g}$ e h, as imagens mostraram aglomerados de $\mathrm{Si}$ em algumas regiões e outras dispersas homogeneamente, o que sugere a formação de estruturas intercaladas. Para as Figuras 2e e f, as cargas estão dispersas homogeneamente sugerindo a formação de estruturas parcialmente esfoliadas. Todas as micrografias estão de acordo com os resultados assinalados pelas análises de DRX e pelas propriedades mecânicas. Comportamento semelhante pode ser encontrado na literatura ${ }^{[12,14,15]}$.

\section{Propriedades térmicas}

As análises de DSC revelaram que o pico endotérmico da fusão apresenta um padrão bimodal do pico endotérmico em relação ao observado para a PA6 pura, sendo que esse padrão é visualizado com mais intensidade para as composições com teores crescentes de argila (Figura 3). A presença do pico endotérmico denominado $\mathrm{T}_{\mathrm{m} 1}$ está relacionada à forma cristalina $\alpha$ da PA6. O surgimento de um segundo pico endotérmico $\left(\mathrm{T}_{\mathrm{m} 2}\right)$ a temperaturas ligeiramente inferiores à $\mathrm{T}_{\mathrm{m} 1}$ está relacionado à fusão cristalina $\left(\mathrm{T}_{\mathrm{m} 2}\right)$ da forma cristalina $\gamma$ da PA6.

$\mathrm{O}$ comportamento para essas temperaturas de fusão $\left(\mathrm{T}_{\mathrm{m} 1}\right.$ e $\mathrm{T}_{\mathrm{m} 2}$ ) sugerem que a forma cristalina $\alpha$ e $\gamma$ coexistem no nanocompósito formado. Esse padrão pode estar associado ao menor grau de empacotamento dos domínios cristalinos da forma $\gamma$. Todavia, a diminuição dos valores de $T_{m}$ dos nanocompósitos pode simplesmente refletir alterações na dimensão lamelar e na distribuição dos cristalitos de PA6 $6^{[14,16,17]}$.

Diversos autores têm relatado o aparecimento de mais um pico endotérmico denominado $\mathrm{T}_{\mathrm{m} 3}$ (Figura 3$)^{[14]}$, o qual foi detectado a partir da adição de 5,0 phr de Claytone 40. Esse padrão pode estar associado à forma $\alpha$ menos estável, a qual é transformado para forma $\alpha$ mais estável causando o aparecimento do pico endotérmico principal $\left(\mathrm{T}_{\mathrm{m} 1}\right)$ através do processo de "recristalização-fusão". Desse modo, pode-se observar que a adição de argila modificada afeta a estrutura e organização dos cristais de PA6 $6^{[14,17]}$.

Quanto à temperatura de cristalização da PA6, fase semicristalina do nanocompósito, pode-se observar na Tabela 2, de modo geral, que a adição da argila contribuiu de forma mínima para redução nos valores de $T_{c}$ em relação à matriz pura de poliamida. Os valores apresentados foram praticamente idênticos entre si. Essa aproximação nos resultados na temperatura de cristalização pode ser atribuída à interação da argila com a fase da poliamida de forma a restringir a mobilidade das cadeias poliméricas ${ }^{[12,14]}$.

Quanto ao grau de cristalinidade (Xc), pode-se observar na Tabela 2 que a adição da argila contribui para leve redução da cristalinidade como consequência da presença da carga, do processo de intercalação e/ou esfoliação e das possíveis interações químicas entre a PA6 e a argila claytone $40^{[18]}$.

\section{Estrutura cristalina}

Os resultados fornecidos pela calorimetria diferencial de varredura sugeriram a formação de cristais $\gamma$ nos nanocompósitos

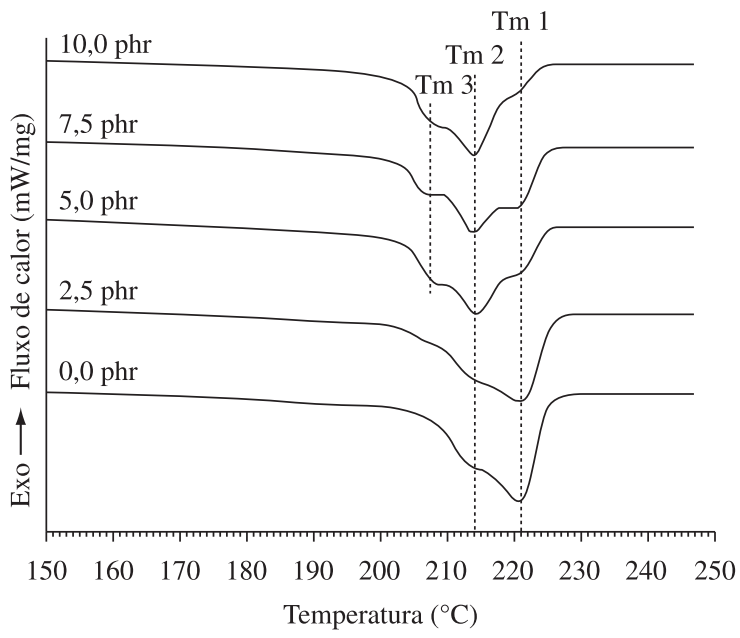

Figura 3. Gráfico das curvas da $2^{\circ}$ corrida de temperatura de fusão $\left(T_{m}\right)$ de calorimetria diferencial de varredura (DSC) da PA6 e seus nanocompósitos com diferentes teores de argila organofílica.

Tabela 2. Propriedades térmicas da PA6 e seus nanocompósitos com diferentes teores de Claytone 40.

\begin{tabular}{cccc}
\hline Clay 40 (phr) & $\mathbf{T c}\left({ }^{\circ} \mathbf{C}\right)$ & $\Delta \mathbf{H m}\left(\mathbf{J . g}^{-1}\right)$ & $\mathbf{X}_{\mathbf{c}}(\boldsymbol{\%})$ \\
\hline 0 & $187,1 \pm 0,2$ & 56,54 & 29,6 \\
2,5 & $185,9 \pm 0,7$ & 51,80 & 27,9 \\
5,0 & $185,5 \pm 0,4$ & 50,02 & 27,5 \\
7,5 & $186,1 \pm 0,8$ & 48,44 & 27,2 \\
10,0 & $186,0 \pm 0,6$ & 45,15 & 26,3 \\
\hline
\end{tabular}

de PA6 e PA6 pura, a qual foi investigada com o auxílio da técnica de DRX, cujos difratogramas são mostrados na Figura 4.

Os difratogramas ilustram uma linha pontilhada atravessando cada amostra no ponto de maior intensidade, caracterizando os picos da forma da estrutura $\gamma$. Esses picos estão geralmente em torno de 21 a $22,5^{\circ}$ correspondendo ao plano de difração (200).

De acordo com a literatura ${ }^{[15,19-22]}$, a PA6 exibe duas formas cristalinas monoclínicas dominantes de cristal denominadas $\alpha$ e $\gamma$. A formação destas duas formas depende principalmente das condições de cristalização ou adição de cargas específicas. A forma da estrutura $\alpha$ é reconhecida como a mais estável termodinamicamente. A característica dos picos por DRX da forma $\alpha$ da PA6 realizada a temperatura ambiente estão localizados em torno de $2 \theta$ igual a 21 e $24^{\circ}$ e indicado como plano de difração (200) e (002)/(202) respectivamente. Por outro lado, a forma da estrutura $\gamma$ mostra picos geralmente em torno de 11,22 e $23^{\circ}$ correspondendo aos planos de difração (020), (001) e (200)/(201) respectivamente.

Os resultados de DRX revelam que a PA6 apresenta um complexo das duas estruturas com maior proporção da forma $\gamma$ do que a forma da estrutura $\alpha_{1}$ e $\alpha_{2}$. Isso pode estar associado às condições de processamento do material na câmara de mistura e moldagem por injeção, seguido de rápido resfriamento do molde ${ }^{[14]}$.

Em relação aos nanocompósitos pode-se observar o mesmo comportamento exibido pela PA6, uma vez que a adição de argila na matriz de PA6 contribuiu para o aparecimento da forma $\gamma$ do cristal.

\section{Propriedades mecânicas}

A Tabela 3 apresenta os resultados dos ensaios de resistência à tração (tensão no escoamento, deformação na ruptura e módulo elástico) da PA6 e seus nanocompósitos com teores crescentes de argila modificada. Os valores representam a média dos resultados 


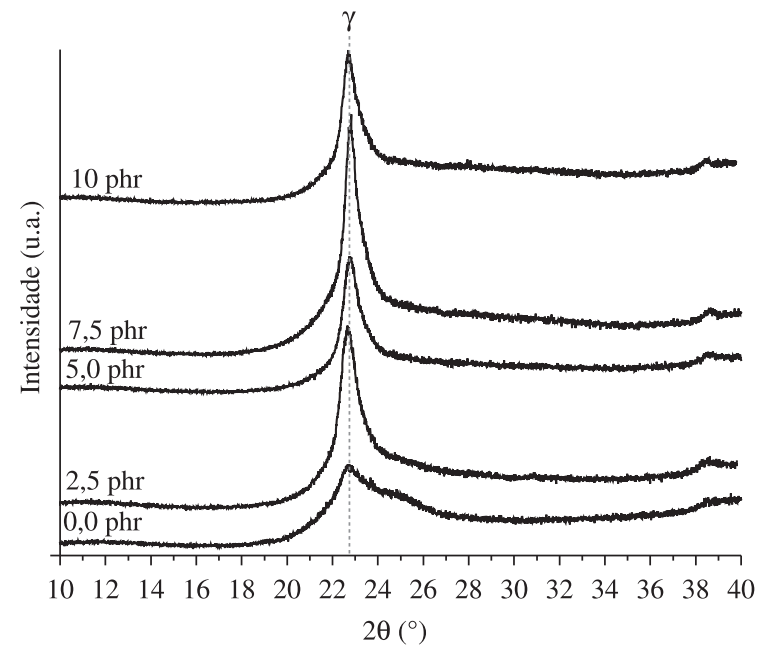

Figura 4. Difratogramas da estrutura cristalina da PA6 e suas composições.

Tabela 3. Propriedades mecânicas da PA6 e dos seus nanocompósitos de PA6 com diferentes teores de argila organofílica.

\begin{tabular}{cccc}
\hline $\begin{array}{c}\text { Clay 40 } \\
\text { (phr) }\end{array}$ & Escoamento & Ruptura & Módulo \\
\cline { 2 - 4 } & $\sigma(\mathbf{M P a})$ & $\varepsilon(\%)$ & $\mathrm{E}(\mathbf{G P a}) *$ \\
\hline 0 & $57,1 \pm 1,3$ & $285,0 \pm 22,8$ & $4,7 \pm 0,8$ \\
2,5 & $63,9 \pm 1,3$ & $300,4 \pm 10,8$ & $4,8 \pm 0,6$ \\
5,0 & $69,0 \pm 0,9$ & $12,3 \pm 2,6$ & $6,5 \pm 0,2$ \\
7,5 & $56,2 \pm 0,9$ & $7,1 \pm 3,5$ & $4,2 \pm 1,1$ \\
10,0 & $47,9 \pm 2,9$ & $11,8 \pm 3,9$ & $4,3 \pm 0,2$ \\
\hline
\end{tabular}

obtidos com seus respectivos desvios padrão conforme o número de corpos de prova previstos na norma utilizada.

A adição de teores superiores a 2,5 phr de argila à PA6 resultou em grande redução da deformação, indicando perda de tenacidade. Nota-se que o nanocompósito com 2,5 phr de claytone 40 apresenta valores bem próximos aos da PA6 pura, sugerindo que tal quantidade de argila é muito pequena para influenciar o comportamento da PA6. Com relação ao escoamento, há um aumento da tensão até o teor de 5,0 phr de Claytone 40.

Isso pode indicar um aumento da rigidez do nanocompósito em função da intercalação/esfoliação das lamelas, conforme assinalado pelas análises de difração de raios X.

De fato, o nanocompósito contendo 5,0 phr de Claytone 40, apresenta maior módulo frente aos demais nanocompósitos e, sua estrutura, conforme revelados pelas análises de DRX podem ser classificados como parcialmente esfoliada.

Alguns trabalhos na literatura encontraram resultados muito semelhantes aos aqui relatado e, atribuíram ao aumento da rigidez e da tensão no escoamento à interação entre a matriz de PA6 e as lamelas de silicato pela formação de ligações de hidrogênio. Outra razão seria a imobilização parcial da fase polimérica confinada entre as lamelas ${ }^{[23]}$. A menor eficiência de reforço observada para teores maiores de argila pode ser atribuída à interação partícula-partícula da argila, favorecendo o seu re-empilhamento ao invés da dispersão, como relatado na referência anterior.

\section{Conclusão}

Nesse trabalho, foi possível obter nanocompósitos com estrutura intercalada e/ou esfoliada, utilizando a técnica de intercalação por fusão. A adição de 2,5 e 5,0 phr de argila na matriz de PA6 indicou a possível formação de estruturas parcialmente esfoliadas, enquanto que a adição de 7,5 e 10 phr resultou na formação de estruturas intercaladas. A introdução da argila organofílica não alterou de modo significativo o grau de cristalinidade, mas favoreceu a formação de cristais $\gamma$. Finalmente, o nanocompósito contendo 5,0 phr da argila Claytone 40 exibiu maiores valores de módulo e tensão no escoamento, provavelmente em função da intercalação/ esfoliação da argila conforme sugerido nas análises de difração de raios $\mathrm{X}$ e microscopia eletrônica.

\section{Agradecimentos}

Os autores agradecem ao auxílio concedido pela CAPES e à Divisão de Catálise e Processos Químicos (DCAP/INT) pelas análises de difração de raios $\mathrm{X}$ na realização desse trabalho.

\section{Referências Bibliográficas}

1. Rodolfo Jr., A. \& Mel, L. H. I. - Polímeros, 19, p.1 (2009).

2. Souza, M. A.; Pessan L. A. \& Rodolfo Jr., A. - Polímeros, 16, p. 257 (2006).

3. Bureau, M. N.; Denault, J.; Cole, K. C. \& Enright, G. D. - Polym. Eng. Sci., 42, p.1897 (2002).

4. Alexandre, M. \& Dubois, P. Mater. Sci. Eng., 28, p.1 (2000).

5. Lebaron, P. C.; Wang, Z. \& Pinnavaia, T. J. - Appl. Clay Sci., 15, p.11 (1999).

6. Ray, S. S. - J. Ind. Eng. Chem., 12, p.811 (2006).

7. Medeiros, V. N.; Araújo, E. D.; Maia, L. F.; Pereira, O. D.; Arimateia, R. F. \& Paz, R. A. - Polímeros, 18, p.302 (2008).

8. Gonella, L. B. - "Nanocompósitos de poliamida 6 com montmorilonitas e xisto", Tese de Doutorado, Universidade Federal do Rio Grande do Sul, Brasil (2007).

9. Chanda, M. \& Roy, S. K. - "Industrial polymers, specialty polymers, and their applications", CRC Press Taylor \& Francis Group, New York (2009).

10. Kusmono; Ishak Z. A. M.; Chow, W. S. \& Takeichi, T. - Eur. Polym. J., 44, p.1023 (2008).

11. López, D. G.; Mitre, I. G.; Fernández, J. F. \& Pastor, J. M. Polymer, 46, p.2758 (2005).

12. Yu, S.; Zhao, J.; Chen, G.; Juay, Y. K. \& Yong, M. S. - J. Mater. Process. Technol., 192-193, p.410 (2007).

13. González, T. V.; Salazar, C. G.; Rosa, J. R. \& González, V. G. - J. Appl. Polym. Sci., 108, p.2923 (2008).

14. Chiu, F.-C.; Lai, S.-M.; Chen, Y.-L. \& Lee, T.-H. - Polymer, 46, p.11600 (2005).

15. Tung, J.; Gupta, R. K.; Simon, G. P.; Edward, G. H. \& Bhattacharya, S. N. - Polymer, 46, p.10405 (2005).

16. González, I.; Eguiazábal, J. I. \& Nazábal, J. Compos. Sci. Technol., 66, p.1833 (2006).

17. Li, T-C.; Ma, J.; Wang, M.; Tjiu, W. C.; Liu, T. \& Huang, W. - J. Appl. Polym. Sci., 103, p.1191 (2007).

18. Mert, M. \& Yilmazer, U. - J. Apll. Polym. Sci., 108, p.3890 (2008).

19. Fornes, T. D. \& Paul, D. R. - Polymer, 44, p.3945 (2003).

20. Zammarano, M.; Bellayer, S.; Gilman, J. W.; Franceschi, M.; Beyer, F. L.; Harris, R. H. \& Meriani, S. - Polymer, 47, p.652 (2006).

21. He, C.; Liu, T.; Tjiu, W. C.; Sue, H.-J. \& Yee, A. F. Macromolecules, 41, p.193 (2008).

22. Fornes, T. D. \& Paul, D. R. Polímeros, 13, p.212 (2003).

23. Aalaie, J.; Khanbabaie, G.; Khoshniyah, R.; Rahmatpour, A. - J. Macromol. Sci.: Part B, 46, p.305 (2007).

Enviado: $30 / 06 / 10$

Reenviado: $11 / 08 / 10$

Aceito: $21 / 08 / 10$

DOI: 10.1590/S0104-14282011005000015 